PROCEEDINGS OF THE

AMERICAN MATHEMATICAL SOCIETY

Volume 129, Number 10, Pages 2881-2888

S 0002-9939(01)05864-6

Article electronically published on February 9, 2001

\title{
PERIODIC POINTS AND NORMAL FAMILIES
}

\author{
DETLEF BARGMANN AND WALTER BERGWEILER
}

(Communicated by Albert Baernstein II)

\begin{abstract}
Let $\mathcal{F}$ be the family of all functions which are holomorphic in some domain and do not have periodic points of some period greater than one there. It is shown that $\mathcal{F}$ is quasinormal, and the sequences in $\mathcal{F}$ which do not have convergent subsequences are characterized. The method also yields a new proof of the result that transcendental entire functions have infinitely many periodic points of all periods greater than one.
\end{abstract}

\section{INTRODUCTION AND MAIN RESULTS}

Let $X, Y$ be sets and let $f: X \rightarrow Y$ be a function. The iterates $f^{n}: X_{n} \rightarrow Y$ are defined by $X_{1}:=X, f^{1}:=f$ and $X_{n}:=f^{-1}\left(X_{n-1} \cap Y\right), f^{n}:=f^{n-1} \circ f$ for $n \in \mathbb{N}, n \geq 2$. Note that $X_{2}=f^{-1}\left(X_{1} \cap Y\right) \subset X=X_{1}$ and thus $X_{n+1} \subset X_{n} \subset X$ for all $n \in \mathbb{N}$.

A point $x \in X$ is called a periodic point of period $n$ of $f$ if $x \in X_{n}$ and $f^{n}(x)=x$, but $f^{m}(x) \neq x$ for $1 \leq m \leq n-1$. A periodic point of period 1 is called a fixed point. The periodic points of period $n$ are thus the fixed points of $f^{n}$ which are not fixed points of $f^{m}$ for any $m$ less than $n$.

The following result is due to P. C. Rosenbloom [16].

Theorem A. Let $f$ be a transcendental entire function and let $n \in \mathbb{N}, n \geq 2$. Then $f^{n}$ has infinitely many fixed points.

This result generalized a result of P. Fatou [11, p. 345] dealing with the case $n=2$. Clearly, polynomials which are not of the form $f(z)=z+c, c \in \mathbb{C}$, and thus their iterates, also have fixed points.

M. Essén and S. Wu [9] proved a corresponding normality criterion, thereby answering a question of L. Yang [18, Problem 8].

Theorem B. Let $D \subset \mathbb{C}$ be a domain and let $\mathcal{G}$ be the family of all holomorphic functions $f: D \rightarrow \mathbb{C}$ for which there exists $n=n(f)>1$ such that $f^{n}$ has no fixed point. Then $\mathcal{G}$ is normal.

The polynomial $p(z)=-z+z^{2}$ has no periodic point of period 2. The following result of I. N. Baker [2] shows that it is essentially the only polynomial of degree greater than one where periodic points of some period are missing.

Theorem C. Let $f$ be a polynomial of degree $d \geq 2$ and let $n \in \mathbb{N}, n \geq 2$. Suppose that $f$ has no periodic point of period $n$. Then $d=n=2$. Moreover, there exists a linear transformation $L$ such that $f(z)=L^{-1}(p(L(z)))$, with $p(z)=-z+z^{2}$.

Received by the editors September 13, 1999 and, in revised form, January 31, 2000.

2000 Mathematics Subject Classification. Primary 30D05, 30D45, 37F10. 
The following generalization of Theorem A was conjectured in [13, Problem 2.20] and proved in [3, Theorem 1] and [4, §1.6, Satz 2].

Theorem D. Let $f$ be a transcendental entire function and let $n \in \mathbb{N}, n \geq 2$. Then $f$ has infinitely many periodic points of period $n$.

Here we shall be concerned with normal family analogues of these results. The method used will also lead to a new proof of Theorem D.

Let $D \subset \mathbb{C}$ be a domain, $m \in \mathbb{N}, m \geq 2$. By $\mathcal{F}_{m}=\mathcal{F}_{m}(D)$ we denote the family of all holomorphic functions $f: D \rightarrow \mathbb{C}$ for which there exists $n=n(f) \geq m$ such that $f$ has no periodic point of period $n$. Clearly $\mathcal{F}_{m+1} \subset \mathcal{F}_{m}$ for $m \geq 2$. In particular, $\mathcal{F}_{3} \subset \mathcal{F}_{2}$.

Since all linear transformations are contained in $\mathcal{F}_{m}$, for all $m \geq 2$, none of the families $\mathcal{F}_{m}$ is normal. Also, if $L$ is a linear transformation and if $p(z)=-z+z^{2}$, then $L^{-1} \circ p \circ L \in \mathcal{F}_{2}$. We shall show that in a certain sense all obstructions to normality arise from these examples.

Recall that a family $\mathcal{F}$ of functions holomorphic in a domain $D$ is called quasinormal (cf. 7, 14, 17) if for each sequence $\left(f_{k}\right)$ in $\mathcal{F}$ there exists a subsequence $\left(f_{k_{j}}\right)$ and a finite set $E \subset D$ such that $\left(f_{k_{j}}\right)$ converges locally uniformly in $D \backslash E$. If the cardinality of the exceptional set $E$ can be bounded independently of the sequence $\left(f_{k}\right)$, and if $q$ is the smallest such bound, then we say that $\mathcal{F}$ is quasinormal of order $q$.

Note that the maximum principle implies that if a sequence $\left(f_{k}\right)$ of functions holomorphic in $D$ converges locally uniformly in $D \backslash\left\{z_{1}, \ldots, z_{q}\right\}$, but not in $D$, then $f_{k} \rightarrow \infty$ in $D \backslash\left\{z_{1}, \ldots, z_{q}\right\}$.

Theorem 1. $\mathcal{F}_{2}(D)$ is quasinormal of order 1 .

To compare this result with Theorem B of Essén and Wu [9] we note that our hypotheses are weaker than theirs; that is, $\mathcal{G} \subset \mathcal{F}_{2}(D)$. As a consequence, we obtain only quasinormality instead of normality.

The following result characterizes the sequences in $\mathcal{F}_{2}(D)$ without convergent subsequences.

Theorem 2. Let $\left(f_{k}\right)$ be a sequence in $\mathcal{F}_{2}(D)$ which does not have a convergent subsequence. Then there exists a compact set $K \subset D$ such that $f_{k} \rightarrow \infty$ locally uniformly in $D \backslash K$. Moreover, for sufficiently large $k$ there exists a simply-connected domain $\Omega_{k} \subset K$ satisfying $\min _{z \in K \backslash \Omega_{k}}\left|f_{k}(z)\right| \rightarrow \infty$ and there exists a quasiconformal homeomorphism $\phi_{k}: \mathbb{C} \rightarrow \mathbb{C}$ such that if $p_{k}$ is defined by $p_{k}(z)=2 z$ for $f_{k} \in \mathcal{F}_{3}$ and $p_{k}(z)=-z+z^{2}$ for $f_{k} \in \mathcal{F}_{2} \backslash \mathcal{F}_{3}$, then $f_{k}(z)=\phi_{k}^{-1}\left(p_{k}\left(\phi_{k}(z)\right)\right)$ for $z \in \Omega_{k}$.

Thus Theorem 2 says that if a sequence $\left(f_{k}\right)$ does not have a convergent subsequence, then $f_{k} \rightarrow \infty$ outside certain small domains, and in these domains $f_{k}$ is quasiconformally conjugate to one of two specific polynomials.

\section{Preliminaries For the Proofs}

As in [3, 9], one of the central tools comes from the Ahlfors theory of covering surfaces. (An account of the Ahlfors theory can be found in [1], [12, Chapter 5] or [15, Chapter XIII].) To state the result we need, let $D \subset \mathbb{C}$ be a domain and let $f: D \rightarrow \mathbb{C}$ be holomorphic. Given a Jordan domain $V \subset \mathbb{C}$, we say that $f$ has an 
island over $V$ if $f^{-1}(V)$ has a component whose closure is contained in $D$. Note that if $U$ is such a component, then $\left.f\right|_{U}: U \rightarrow V$ is a proper map.

Lemma 1. Let $D \subset \mathbb{C}$ be a domain and let $D_{1}, D_{2} \subset \mathbb{C}$ be Jordan domains with $\overline{D_{1}} \cap \overline{D_{2}}=\emptyset$. Let $\mathcal{F}$ be a family of functions holomorphic in $D$ which is not normal. Then there exists a function $f \in \mathcal{F}$ which has an island over $D_{1}$ or $D_{2}$.

Lemma 1 follows from [12, Theorem 5.5] (applied with a domain $D_{3}$ containing $\infty$ ) and [12, Theorem 6.6]. For a different proof of Lemma 1 see [5, §5.1].

Another important concept used in [3, 9], and again used here, is that of a polynomial-like map. By definition, if $U, V \subset \mathbb{C}$ are bounded, simply-connected domains with $\bar{U} \subset V$, and if $f: U \rightarrow V$ is a proper map (of degree $d$ ), then the triple $(f, U, V)$ is called a polynomial-like map (of degree $d$ ). The fundamental result about polynomial-like maps is the following (see [6] Theorem VI.1.1] or [8] Theorem 1]).

Lemma 2. Let $(f, U, V)$ be a polynomial-like map of degree $d$. Then there exists a polynomial $p$ of degree $d$ and a quasiconformal map $\phi: \mathbb{C} \rightarrow \mathbb{C}$ such that $f(z)=$ $\phi^{-1}(p(\phi(z)))$ for all $z \in U$. Moreover, $\phi(U)$ contains the filled Julia set of $p$ and thus, in particular, all periodic points of $p$.

If $d=1$, then $p$ has the form $p(z)=a z+b$ with $a, b \in \mathbb{C}$. It is not difficult to see that $|a|>1$. With $L(z)=z+b /(a-1)$ we have $p(z)=L^{-1}(a L(z))$. With $\psi(z)=z|z|^{\alpha} e^{i \beta \log |z|}$ where $\alpha=\log 2 / \log |a|-1$ and $\beta=-\arg a / \log |a|$ we have $a z=\psi^{-1}(2 \psi(z))$. Replacing $\phi$ by $\psi \circ L \circ \phi$ we obtain the following:

Remark. If $d=1$ in Lemma 2, then we can choose $p(z)=2 z$ there.

A simple consequence of Lemma 2 (which, however, can also be proved directly) is the following result.

Lemma 3. Every polynomial-like map has a fixed point.

Let $f: D \rightarrow \mathbb{C}$ be holomorphic and let $U, V \subset \mathbb{C}$ be Jordan domains. It will be convenient to use the notation $U \stackrel{f}{\rightsquigarrow} V$ if $\left.f\right|_{D \cap U}$ has an island over $V$. Note that if $U \stackrel{f}{\rightsquigarrow} V$ and $V \stackrel{g}{\sim} W$, then $U \stackrel{g \circ f}{\sim} W$. Also, if $V \stackrel{f}{\sim} V$, then there exists $U \subset V$ such that $(f, U, V)$ is a polynomial-like map. Lemma 3 thus yields the following result.

Lemma 4. If $V \stackrel{f}{\rightsquigarrow} V$, then $f$ has a fixed point in $V$.

We shall use some elementary concepts from graph theory. We mention that graph theoretic arguments like the ones below are also implicit in 9], even though they are not formalized this way.

For a set $V$ and a set $E \subset V \times V$ we call the pair $G=(V, E)$ a digraph. The elements of $V$ are called vertices and those of $E$ are called edges. (Note that in contrast to the usual terminology we allow loops; that is, edges $e$ of the form $e=(v, v)$ with $v \in V$.)

Let $n \in \mathbb{N}$ and $w=\left(v_{0}, v_{1}, \ldots, v_{n}\right) \in V^{n+1}$. Then $w$ is called a closed walk of length $n$ if $\left(v_{k-1}, v_{k}\right) \in E$ for $k \in\{1, \ldots, n\}$ and $v_{0}=v_{n}$. Note that we have not excluded the case that $v_{j}=v_{k}$ for $j, k \in\{1, \ldots, n\}, j \neq k$. We call a closed walk $w=\left(v_{0}, v_{1}, \ldots, v_{n}\right)$ primitive if there do not exist $p \in \mathbb{N}, 1 \leq p<n$, such that $p \mid n$ and $v_{j}=v_{k}$ for all $j, k \in\{1, \ldots, n\}$ satisfying $p \mid(j-k)$. A primitive closed walk is thus a closed walk which is not obtained by running through a closed walk 
of smaller length several times. Finally recall that the outdegree of a vertex $v$ is defined to be the cardinality of $\{u \in V:(v, u) \in E\}$.

Lemma 5. Let $n \in \mathbb{N}$ and let $G=(V, E)$ be a digraph. Suppose that there exist $u, v \in V$ such that $u \neq v$ and $\{(v, v),(u, v),(v, u)\} \subset E$. Then $G$ contains a primitive closed walk of length $n$.

Proof. Since $(v, v)$ is closed walk of length 1 we may assume that $n \geq 2$. Let $v_{0}:=v_{n}:=u$ and $v_{j}:=v$ for $j \in\{1, \ldots, n-1\}$. Then $\left(v_{0}, \ldots, v_{n}\right)$ is a primitive closed walk of length $n$ in $G$.

Remark. The hypotheses of Lemma 5 are satisfied if the cardinality of $V$ is at least 2 and $G$ is the complete digraph; that is, if $E=V \times V$.

Lemma 6. Let $q, n \in \mathbb{N}, q \geq 4, n \geq 2$. Let $G=(V, E)$ be a digraph with $q$ vertices such that the outdegree of each vertex is at least $q-1$. Then $G$ contains a primitive closed walk of length $n$.

Proof. Suppose first that there exists $v \in V$ such that $(v, v) \in E$. Since the outdegree of $v$ is at least 3 there exist $v_{1}, v_{2} \in V \backslash\{v\}$ such that $v_{1} \neq v_{2}$ and $\left(v, v_{j}\right) \in$ $E$ for $j \in\{1,2\}$. If $\left(v_{1}, v\right) \in E$ or $\left(v_{2}, v\right) \in E$, then we can apply Lemma 5 and the conclusion follows. If $\left(v_{1}, v\right) \notin E$ and $\left(v_{2}, v\right) \notin E$, then $\left\{\left(v_{1}, v_{1}\right),\left(v_{1}, v_{2}\right),\left(v_{2}, v_{1}\right)\right\} \subset$ $E$, and again the conclusion follows from Lemma [5]

Suppose now that $(v, v) \notin E$ for all $v \in V$. Then $E=(V \times V) \backslash\{(v, v): v \in V\}$. Let $v \in V$ and $v_{0}:=v_{n}:=v$. For $j \in\{1, \ldots, n-1\}$ we choose $v_{j} \in V \backslash\{v\}$ inductively such that $v_{j} \neq v_{j-1}$ for $j \in\{1, \ldots, n-1\}$. Then $\left(v_{0}, \ldots, v_{n}\right)$ is a primitive closed walk of length $n$ in $G$.

Given pairwise disjoint Jordan domains $D_{1}, \ldots, D_{q} \subset \mathbb{C}$ and a holomorphic function $f$ we shall apply Lemmas [5 and [6 to the digraph $G\left(f,\left\{D_{j}\right\}_{j=1}^{q}\right)$ whose vertex set $V$ and edge set $E$ are given by $V=\left\{D_{j}: j=1, \ldots, q\right\}$ and $E=$ $\left\{\left(D_{j}, D_{k}\right) \in V \times V: D_{j} \stackrel{f}{\rightsquigarrow} D_{k}\right\}$.

Lemma 7. If $G\left(f,\left\{D_{j}\right\}_{j=1}^{q}\right)$ contains a primitive closed walk of length $n$, then $f$ has a periodic point of period $n$ in each $D_{j}$ belonging to the walk.

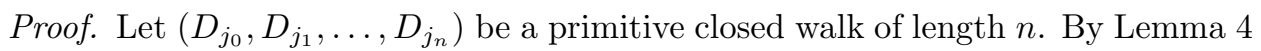
and the remarks made before Lemma 4 there exists a fixed point $z_{0} \in D_{j_{0}}$ of $f^{n}$ such that $f^{k}\left(z_{0}\right) \in D_{j_{k}}$ for $k \in\{1, \ldots, n-1\}$. It follows from the definition of primitivity that $z_{0}$ has period $n$.

\section{Proof of Theorems 1 AND 2}

Proof of Theorem [1. We may assume that $D$ is bounded. Let $q \in \mathbb{N}$, let $z_{1}, \ldots, z_{q} \in$ $D$ be distinct and let $\left(f_{k}\right)$ be a sequence in $\mathcal{F}_{2}$ such that no subsequence of $\left(f_{k}\right)$ is normal in a neighborhood of some $z_{j}$. We will show that $q=1$. This implies that $\mathcal{F}_{2}(D)$ is quasinormal, and in fact quasinormal of order at most 1 .

For $j \in\{1, \ldots, q\}$ let $D_{j}$ be a disk around $z_{j}$ such that $\overline{D_{j}} \subset D$ and $\overline{D_{i}} \cap \overline{D_{j}}=\emptyset$ for $i \neq j$. We consider the digraphs $G_{k}=G\left(f_{k},\left\{D_{j}\right\}_{j=1}^{q}\right)$.

First we show that $q<4$. We assume that this is not the case. Lemma 1 implies that the outdegree of each vertex of $G_{k}$ is at least $q-1$ if $k$ is sufficiently large. Lemma 6 implies that for these $k$ there is a primitive closed walk of length $n$ for 
each $n \geq 2$. From Lemma 7 we deduce that $f_{k}$ has a periodic point of period $n$ for each $n \geq 2$, provided $k$ is large enough. This is a contradiction. Hence $q<4$.

We may now assume that $q<4$ has been chosen maximal so that $\left(f_{k}\right)$ is normal in $D \backslash\left\{z_{1}, \ldots, z_{q}\right\}$. It follows that $f_{k} \rightarrow \infty$ locally uniformly in $D \backslash\left\{z_{1}, \ldots, z_{q}\right\}$. This implies that $f_{k}\left(\partial D_{i}\right) \cap \overline{D_{j}}=\emptyset$ for $i, j \in\{1, \ldots, q\}$ and large $k$. Since no subsequence of $\left(f_{k}\right)$ is normal, we also have $f_{k}\left(D_{i}\right) \cap D_{j} \neq \emptyset$ for $i, j \in\{1, \ldots, q\}$ and large $k$. It follows that $D_{i} \stackrel{f_{k}}{\rightsquigarrow} D_{j}$ for $i, j \in\{1, \ldots, q\}$ and large $k$. Hence $G_{k}$ is the complete digraph if $k$ is sufficiently large. On the other hand, since $f_{k} \in \mathcal{F}_{2}$ for all $k \in \mathbb{N}$, there exists $n_{k} \in \mathbb{N}, n_{k} \geq 2$, such that $f$ has no periodic point of period $n_{k}$. Thus $G_{k}$ does not contain a primitive closed walk of length $n_{k}$ by Lemma 7 Lemma 5 and the remark following it now show that $q=1$.

Proof of Theorem Q Again we may assume that $D$ is bounded. Since no subsequence of $\left(f_{k}\right)$ converges to $\infty$ there exist $M, \delta>0$ and a sequence $\left(z_{k}\right)$ in $D$ such that $\operatorname{dist}\left(z_{k}, \partial D\right) \geq \delta$ and $\left|f_{k}\left(z_{k}\right)\right| \leq M$ for all $k$. We define $H_{k}:=\{z \in \mathbb{C}:$ $\left.\left|z-z_{k}\right|<\delta / 2\right\}$ and $m_{k}:=\min _{z \in \partial H_{k}}\left|f_{k}(z)\right|$. Choose $\zeta_{k} \in \partial H_{k}$ with $m_{k}=\left|f_{k}\left(\zeta_{k}\right)\right|$.

Suppose that $\left(m_{k}\right)$ has a bounded subsequence $\left(m_{k_{j}}\right)$. Passing to further subsequences if necessary, we may assume that $z_{k_{j}} \rightarrow z_{0} \in D$ and $\zeta_{k_{j}} \rightarrow \zeta_{0} \in D$. Since $\left(f_{k_{j}}\right)$ is quasinormal of order 1 by Theorem 1 , we may also assume that $\left(f_{k_{j}}\right)$ converges in $D \backslash\left\{z_{1}\right\}$ for some $z_{1} \in D$. As noted before Theorem 1, we actually have $f_{k_{j}} \rightarrow \infty$ in $D \backslash\left\{z_{1}\right\}$. Since $\left|f_{k_{j}}\left(z_{k_{j}}\right)\right|<M$ this implies that $z_{1}=z_{0}$ and hence that $m_{k_{j}}=\left|f_{k_{j}}\left(\zeta_{k_{j}}\right)\right| \rightarrow \infty$. This is a contradiction. Hence $\left(m_{k}\right)$ does not have a bounded subsequence; that is, $m_{k} \rightarrow \infty$.

Let $\Delta_{k}:=\left\{z \in \mathbb{C}:|z|<m_{k} / 2\right\}$. Then $f_{k}\left(\partial H_{k}\right) \cap \overline{\Delta_{k}}=\emptyset$ for all $k$. For large $k$ we have $m_{k}>2 M$ and thus $f_{k}\left(z_{k}\right) \in \Delta_{k}$ so that $f_{k}\left(H_{k}\right) \cap \Delta_{k} \neq \emptyset$. Hence $H_{k} \stackrel{f_{k}}{\rightsquigarrow} \Delta_{k}$ for large $k$. Since $D$ is bounded and $m_{k} \rightarrow \infty$ we also have $\overline{H_{k}} \subset \Delta_{k}$ for large $k$. For these $k$ we put $\Omega_{k}:=f_{k}^{-1}\left(\Delta_{k}\right) \cap H_{k}$. Let $D_{1}, \ldots, D_{q}$ be components of $\Omega_{k}$. Then $G_{k}=G\left(f_{k},\left\{D_{j}\right\}_{j=1}^{q}\right)$ is the complete graph, and thus $q=1$ by Lemmas 5 and 7 Hence $\Omega_{k}$ is connected and, by the maximum principle, even simply-connected.

Thus $\left(f, \Omega_{k}, \Delta_{k}\right)$ is a polynomial-like map. By Lemma 2 there exists a quasiconformal map $\phi_{k}: \mathbb{C} \rightarrow \mathbb{C}$ and a polynomial $p_{k}$ such that $f_{k}(z)=\phi_{k}^{-1}\left(p_{k}\left(\phi_{k}(z)\right)\right)$ for $z \in \Omega_{k}$. Now $f_{k} \in \mathcal{F}_{m}(D)$ implies that $p_{k} \in \mathcal{F}_{m}(\mathbb{C})$. From Theorem $\mathrm{C}$ we thus deduce that if $f_{k} \in \mathcal{F}_{3}(D)$, then $p_{k}$ has degree 1. By the remark following Lemma[2 we may thus assume that $p_{k}(z)=2 z$ in this case. From Theorem $\mathrm{C}$ we can also deduce that if $f_{k} \in \mathcal{F}_{2}(D) \backslash \mathcal{F}_{3}(D)$, then $p_{k}$ has the form $p_{k}(z)=L_{k}^{-1}\left(p\left(L_{k}(z)\right)\right.$, with $p(z)=-z+z^{2}$ and a linear transformation $L_{k}$. Replacing $\phi_{k}$ by $L_{k} \circ \phi_{k}$ we obtain the conclusion.

\section{A proof of Theorem D}

Let $\left(a_{k}\right)$ be a sequence tending to $\infty$ such that $\left(f\left(a_{k}\right)\right)$ is bounded. Define $f_{k}: \mathbb{C} \rightarrow \mathbb{C}$ by $f_{k}(z)=f\left(a_{k} z\right) / a_{k}$. Then $f_{k}(0) \rightarrow 0$ and $f_{k}(1) \rightarrow 0$.

Suppose now that $f$ has only finitely many periodic points of period $n$. Theorem 1 implies that $\left(f_{k}\right)$ is quasinormal of order 1 in $\mathbb{C} \backslash\{0\}$. It is not difficult to show that no subsequence of $\left(f_{k}\right)$ is normal at 0 , and this implies that $f_{k} \rightarrow \infty$ in $\mathbb{C} \backslash\{0,1\}$.

For sufficiently large $k$ we have $\left|f_{k}(0)\right|<2,\left|f_{k}(1)\right|<2, \min _{|z|=1 / 2}\left|f_{k}(z)\right|>2$ and $\min _{|z-1|=1 / 2}\left|f_{k}(z)\right|>2$. This implies that there are components $D_{0}$ and $D_{1}$ of $f_{k}^{-1}(\{z \in \mathbb{C}:|z|<2\})$ satisfying $0 \in D_{0} \subset\left\{z \in \mathbb{C}:|z|<\frac{1}{2}\right\}$ and 
$1 \in D_{1} \subset\left\{z \in \mathbb{C}:|z-1|<\frac{1}{2}\right\}$. It follows that $G\left(f_{k},\left\{D_{j}\right\}_{j=0}^{1}\right)$ is the complete graph. From Lemmas 5 and 7 we deduce that $f_{k}$ has a periodic point of period $n$ in $D_{1}$, and thus $f$ has a periodic point of period $n$ in $\left\{z \in \mathbb{C}:\left|z-a_{k}\right|<\frac{1}{2}\left|a_{k}\right|\right\}$ for large $k$. This is a contradiction.

\section{REPELLING PERIODIC POINTS}

Let $z_{0} \in \mathbb{C}$ be a periodic point of period $n$ of the holomorphic function $f$. Then $z_{0}$ is called repelling if $\left|\left(f^{n}\right)^{\prime}\left(z_{0}\right)\right|>1$.

M. Essén and S. Wu [10, Theorem 1] have generalized their Theorem B as follows.

Theorem E. Let $D \subset \mathbb{C}$ be a domain and let $\mathcal{G}$ be the family of all holomorphic functions $f: D \rightarrow \mathbb{C}$ for which there exists $n=n(f)>1$ such that $f^{n}$ has no repelling fixed point. Then $\mathcal{G}$ is normal.

Theorems $\mathrm{C}$ and $\mathrm{D}$ also have generalizations dealing with repelling periodic points.

Theorem F. Let $f$ be a polynomial of degree $d \geq 2$ and let $n \in \mathbb{N}$. Suppose that $f$ has no repelling periodic point of period $n$. Then one of the following cases holds:

(i) $n=1, d \geq 2$,

(ii) $n=2, d=2$,

(iii) $n=2, d=3$,

(iv) $n=2, d=4$,

(v) $n=3, d=2$.

Theorem G. Let $f$ be a transcendental entire function and let $n \in \mathbb{N}, n \geq 2$. Then $f$ has infinitely many repelling periodic points of period $n$.

Theorem $\mathrm{F}$ is proved in [4, $\S 1.4$, Satz 1] while Theorem $\mathrm{G}$ can be found in [3] Theorem 1] and [4, §1.6, Satz 2]. Examples in [4 \$1.4] show that each of the five exceptional cases listed in Theorem $\mathrm{F}$ does occur.

Here we give the following result.

Theorem 3. Let $D \subset \mathbb{C}$ be a domain and let $\mathcal{F}$ be the family of all holomorphic functions $f: D \rightarrow \mathbb{C}$ for which there exists $n=n(f)>1$ such that $f$ has no repelling periodic point of period $n$. Then $\mathcal{F}$ is quasinormal.

It seems possible to describe the non-normal sequences in $\mathcal{F}$ similarly as in Theorem 2 but we omit this here.

We only sketch the proof of Theorem 3. Given a holomorphic function $f: D \rightarrow \mathbb{C}$ and a Jordan domain $V \subset \mathbb{C}$, we say that $f$ has a simple island over $V$ if $f^{-1}(V)$ has a component $U$ with $\bar{U} \subset D$ such that $\left.f\right|_{U}: U \rightarrow V$ is bijective. If, in addition, $\bar{U} \subset V$, then $(f, U, V)$ is a polynomial-like map of degree 1 and, as already mentioned after Lemma 2 (and also easily proved directly), $f$ has a repelling fixed point in $U$ in this case.

Instead of Lemma 1 we now use the following result from the Ahlfors theory.

Lemma 8. Let $D \subset \mathbb{C}$ be a domain and let $D_{1}, D_{2}, D_{3}$ be Jordan domains with pairwise disjoint closures. Let $\mathcal{F}$ be a family of functions holomorphic in $D$ which is not normal. Then there exists a function $f \in \mathcal{F}$ which has a simple island over $D_{1}, D_{2}$ or $D_{3}$. 
We also replace Lemma 6 by the following one.

Lemma 9. Let $q, n \in \mathbb{N}, q \geq 6, n \geq 2$. Let $G$ be a digraph with $q$ vertices such that the outdegree of each vertex is at least $q-2$. Then $G$ contains a primitive closed walk of length $n$.

With these modifications, the proof of Theorem 3 proceeds similar to that of Theorem 1. In fact one obtains that $\mathcal{F}$ is quasinormal of order less than 6 . We omit the details.

Essén and $\mathrm{Wu}[9, \S 4$, Remark 1] have raised the question whether the conclusion of Theorem B holds if one replaces "holomorphic" by "meromorphic" in the definition of $\mathcal{G}$. They show [9, $\S 4$, Remark 2] that the resulting family $\mathcal{G}$ is still quasinormal of order less than 17.

Similarly we may replace "holomorphic" by "meromorphic" in Theorem 3 . To see this we note that in the meromorphic case an analogue of Lemma 8 holds if we take five domains $D_{j}$ instead of three domains - this is the celebrated "Ahlfors five islands theorem". And if we assume in Lemma 9 only that the outdegree of each vertex is at least $q-4$, then the conclusion still holds if $q$ is sufficiently large.

\section{ACKNOWLEDGMENT}

We thank the referee for a number of valuable suggestions.

\section{REFERENCES}

[1] L. V. Ahlfors, Zur Theorie der Überlagerungsflächen, Acta Math. 65 (1935), 157-194, and Collected Papers, Birkhäuser, Boston, Basel, Stuttgart, 1982, Vol. I, pp. 214-251. MR 84k:01066a

[2] I. N. Baker, Fixpoints of polynomials and rational functions, J. London Math. Soc. 39 (1964), 615-622. MR 30:230

[3] W. Bergweiler, Periodic points of entire functions: proof of a conjecture of Baker, Complex Variables Theory Appl. 17 (1991), 57-72. MR 92m:30048

[4] _ Periodische Punkte bei der Iteration ganzer Funktionen. Habilitationsschrift, Rheinisch-Westfälische Techn. Hochsch., Aachen 1991.

[5] A A new proof of the Ahlfors five islands theorem, J. Analyse Math. 76 (1998), 337-347. MR 99m:30032

[6] L. Carleson and T. W. Gamelin, Complex Dynamics, Springer, New York, Berlin, Heidelberg, 1993. MR 94h:30033

[7] C.-T. Chuang, Normal Families of Meromorphic Functions, World Scientific, Singapore, 1993.

[8] A. Douady and J. H. Hubbard, On the dynamics of polynomial-like mappings, Ann. Sci. École Norm. Sup. (4) 18 (1985), 287-343. MR 87f:58083

[9] M. Essén and S. Wu, Fix-points and a normal family of analytic functions, Complex Variables Theory Appl. 37 (1998), 171-178. MR 99m:30069

[10] Repulsive fixpoints of analytic functions with applications to complex dynamics, $J$. London Math. Soc. (2) 62 (2000), 139-148.

[11] P. Fatou, Sur l'itération des fonctions transcendantes entières, Acta Math. 47 (1926), 337-360.

[12] W. K. Hayman, Meromorphic Functions, Clarendon Press, Oxford, 1964. MR 29:1337

[13] _ Research Problems in Function Theory, Athlone Press, London, 1967. MR 36:359

[14] P. Montel, Leçons sur les familles normales des fonctions analytiques et leurs applications, Gauthier-Villars, Paris, 1927.

[15] R. Nevanlinna, Eindeutige analytische Funktionen, Springer, Berlin, Göttingen, Heidelberg, 1953. MR 15:208c

[16] P. C. Rosenbloom, L'itération des fonctions entières, C. R. Acad. Sci. Paris 227 (1948), 382-383. MR 10:187a 
[17] J. L. Schiff, Normal Families, Springer, New York, Berlin, Heidelberg, 1993. MR 94f:30046

[18] L. Yang, Some recent results and problems in the theory of value-distribution, in Proceedings of the Symposium on Value Distribution Theory in Several Complex Variables, (W. Stoll, ed.), Univ. of Notre Dame Press, Notre Dame Math. Lect. 12 (1992), 157-171. MR 94i:30029

Mathematisches Seminar, Christian-Albrechts-Universität Zu Kiel, Ludewig-MeynStr. 4, D-24098 Kiel, Germany

E-mail address: bargmann@math.uni-kiel.de

Mathematisches Seminar, Christian-Albrechts-Universität zu Kiel, Ludewig-MeynStr. 4, D-24098 Kiel, Germany

E-mail address: bergweiler@math.uni-kiel.de 